\title{
Minimizing Adverse Effects of Cerenkov Radiation Induced Photodynamic Therapy With Transformable Photosensitizer-loaded Nanovesicles
}

\section{Ruijie Qian}

Wuhan Union Hospital

\section{Kun Wang}

Wuhan Union Hospital

\section{Yawen Guo}

Tongji Hospital of Tongji Medical College of Huazhong University of Science and Technology

Hongyan Li

Wuhan Union Hospital

\section{Ziyang Zhu}

Wuhan Union Hospital

\section{Xiaojuan Huang}

Zhejiang University School of Medicine Sir Run Run Shaw Hospital

\section{Chengpeng Gong}

Wuhan Union Hospital

Yu Gao

Wuhan Union Hospital

\section{Rong Guo}

Wuhan Union Hospital

\section{Biao Yang}

Wuhan Union Hospital

\section{Chenyang Wang}

Wuhan Union Hospital

\section{Dawei Jiang}

Wuhan Union Hospital

\section{Xiaoli Lan}

Wuhan Union Hospital

\section{Rui An}

Wuhan Union Hospital

Zairong Gao ( $\nabla$ gaobonn@163.com )

Wuhan Union Hospital https://orcid.org/0000-0002-8367-2659 


\section{Research Article}

Keywords: Cerenkov radiation, exosome coating, nuclear medicine, nanomedicine, photodynamic therapy, synergistic therapy, breast cancer

Posted Date: December 17th, 2021

DOI: https://doi.org/10.21203/rs.3.rs-1135369/v1

License: (c) (i) This work is licensed under a Creative Commons Attribution 4.0 International License.

Read Full License 


\section{Abstract}

\section{Background}

Photodynamic therapy (PDT) is a promising antitumor strategy with fewer adverse effects and higher selectivity than conventional therapies. Recently, a series of reports have suggested that PDT (CR-PDT) induced by Cerenkov radiation (CR) has deeper tissue penetration than traditional PDT. While the combination strategy by coupling radionuclides with photosensitizers might cause severe side effects.

Methods

We designed tumor-targeting nanoparticles ( 131 I-EM@ALA) by loading 5-Aminolevulinic acid (ALA) into 131 Hlabeled exosome mimetic (EM) to achieve combined antitumor therapy. In addition to the role of radiotherapy, 131 I can also serve as an internal light source for its Cerenkov radiation (CR).

\section{Results}

The drug-loaded nanoparticles could effectively target tumors as confirmed by confocal imaging, flow cytometry, and small animal fluorescence imaging. The in vitro and in vivo experiments demonstrated that 131 I-EM@ALA produced a promising antitumor effect by synergizing radiotherapy and CR-PDT. The nanoparticles killed tumor cells by inducing DNA damage and activating the lysosome-mitochondrial pathways. During the treatment, there were no obvious abnormalities found in hematology analyses, blood biochemistry, and histological examinations.

\section{Conclusions}

We successfully engineered nanocarrier coloaded with radionuclide $131 \mathrm{I}$ and a photosensitizer precursor for combinational radiotherapy and PDT in treatment of breast cancer.

\section{Background}

Photodynamic therapy (PDT) is already a clinically approved and promising therapeutic modality to treat the neoplastic and non-malignant diseases (1). PDT means irradiating photosensitizers (PSs) with light of specific wavelength triggers the generation of reactive oxygen species (ROS), leading to cancer cell death(2). However, PDT was confined to superficial tissues due to the rapid attenuation of light in tissue and the concomitant tissue penetration limitation (3).Several strategies have been designed to solve this problem including near-infrared light, self-luminescence, X-ray radiation, and Cerenkov radiation (CR)(4-7). Among them, nearby photosensitizers are activated by $\mathrm{CR}$ produced from radionuclides to generate detrimental ROS, which has been a promising type of PDT broken the depth dependency $(5,8,9)$. Cerenkov radiation-induced PDT (CR-PDT) means Cerenkov luminescence (CL) produced from radionuclides activates photosensitizers to produce ROS. This phenomenon is also known as Cerenkov resonance energy transfer $(\mathrm{CRET})(3,10)$. However, several problems remain to be solved. When the radionuclide and photosensitizer were administered separately, the CR interaction may be weaken and the 
therapeutic effect of the CR-PDT may be decreased (11); While the combination strategy by coupling radionuclide with photosensitizer might continuously generate ROS during its blood circulation, which makes this modality effectively a normal chemotherapeutic agent(12). Thus, the development of a CRPDT strategy with high-efficacy and tumor accumulation while minimizing adverse effects is of major significance but remains challenging (12).

Exosomes are considered to be promising delivery vehicles due to the excellent biocompatibility, drugcarrying capacity, and tumor targeting ability $(13,14)$. However, there are big hurdles for therapeutic use of exosomes, primarily due to their low yield from cell cultures $(15,16)$. In view of the low yield exosome production, several studies have demonstrated that exosome-mimetics (EM) could substitute exosomes perfectly $(17,18)$. EMs, which are prepared by the serial extrusion of cells, have cell membranes and sizes similar to those of exosomes, higher yield, and the same powerful tumor targeting ability (19-22). To further improve the efficiency of drug delivery, FDA-approved 5-Aminolevulinic acid (ALA) was employed to optimize the administration agents. In the heme biosynthesis pathway, ALA is a natural precursor of protoporphyrin IX (PpIX) which is a commonly used photosensitizer in clinical PDT(23). Abundant mitochondria are necessary for the conversion of ALA to PpIX. Thus, at tumor site, CR from ${ }^{131}$ I decay activated PpIX to produce ROS, killing tumor cells. But in normal tissues, ALA hardly converts to PpIX『resulting in little damage. Besides, the dominant emission of CR is in the ultraviolet and blue end of the visible spectrum(15), which ideally responds to ultraviolet and blue wavelengths-responsive PSs such

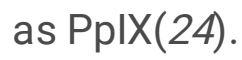

Taken together, we designed a novel modality to optimize the therapeutic effect of CR-PDT to reduce side effects and improve the antitumor efficacy of CR-PDT. ${ }^{131} \mathrm{I}$ is efficiently labeled on ALA-loaded EM(EM@ALA) with chloramine-T method ( ${ }^{131}$ I-EM@ALA). After intravenous (i.v.) administration, the ${ }^{131}$ IEM@ALA could accumulate in tumors because of the enhanced permeability and retention (EPR) effect and homologous targeting characteristics(20-22). Due to the abundant mitochondria in the tumor site, ALA was transformed into active photosensitizer PpIX to generate ROS under CR stimulation, the combination therapy of ${ }^{131} \mathrm{I}$ radiation therapy (RT) and CR-PDT was expected to achieve stronger antitumor effect. While the relative low accumulation of PpIX in normal tissues avoided the detonator payload to minimize the adverse effects from CR-PDT. Overall, the local generation of PpIX in tumor tissues could significantly enhance the tumor therapy effect and reduce adverse effects of CR-PDT, providing a promising strategy for CR-PDT tumor therapy.

\section{Materials}

\section{Preparation and characterization of EM@ALA}

Experiments were performed with mouse breast cancer cell line 4T1, which was maintained in 1640 (Gibco) containing 10\% fetal bovine serum (FBS, Gibco), 10,000 units of penicillin and $10 \mathrm{mg} / \mathrm{mL}$ of streptomycin. Cells were cultivated at $37^{\circ} \mathrm{C}$ and 5\% CO2. EM@ALA was prepared according to the previous report (25). 4T1 cancer cell line was a generous gift from oncology department, Union Hospital, 
Tongji Medical College, Huazhong University of Science and Technology. 4T1 cancer cells were collected by trypsin, resuspended by hypotonic solution (PBS: $\mathrm{ddH} 2 \mathrm{O}=1: 3$ ), and disrupted at $4^{\circ} \mathrm{C}$ for 2 hours. Afterwards, the broken $4 \mathrm{~T} 1$ cells were centrifuged at $700 \mathrm{~g}$ for 10 mins at $4^{\circ} \mathrm{C}$ and the collected sediment was resuspended and centrifuged again at $14,000 \mathrm{~g}$ for $30 \mathrm{mins}$. After that, the $4 \mathrm{~T} 1$ cell membrane and ALA solution were passed through a 0.4 um polycarbonate membrane by extruded physically for 11 times.

The result of particle size and zeta potential distributions were gained by using a Zetasizer Nano ZS (Malvern Instruments, Malvern, UK). EM@ALA solutions were stored at $4^{\circ} \mathrm{C}$ for stability analysis. A transmission electron microscope (TEM; JEM-2010 ES500 W, Japan) operating at $200 \mathrm{keV}$ was used to show the morphology of EM@ALA. Representative protein of EM@ALA was analyzed by western blot (WB). The absorption properties of samples was revealed by UV-vis spectrometry (Synergy 2, BioTek Instruments Inc., USA).

\section{The preparation of ${ }^{131}$ I-EM@ALA}

${ }^{131} \mathrm{I}$-EM@ALA was prepared by chloramine T method(26). Briefly, $40 \mu \mathrm{l}(30 \mathrm{mci} / \mathrm{ml}){ }^{131} \mathrm{I}$ (Gaotong, Chengdu, China) solution was added to $50 \mu \mathrm{l}$ EM@ALA solution, then $15 \mu \mathrm{l} 25 \mathrm{mg} / \mathrm{ml}$ chloramine $T$ solution was added to the mixtures and vortexed for $90 \mathrm{~s}$. Then $5 \mu 100 \mathrm{mg} / \mathrm{ml}$ sodium pyrosulfite solution was added to terminate the reaction. The labeling efficiency and stability were measured by thin layer chromatography (TLC). The instant thin-layer chromatography-silica gel(iTLC-SG) was used as the medium and normal saline as the developing agent. The stability of labeling rate in fetal bovine serum (FBS) and PBS within 48 hours was measured by this method.

\section{Cell viability experiment}

CCK-8 assay was used to evaluate the cytotoxicity effect of ${ }^{131}$ I-EM@ALA. 4T1 cells were seeded in 96well plates at a density of 6,000 cells/well and cultured for 24 hours. ${ }^{131}$ I-EM@ALA, ${ }^{131}$ I-CM, EM@ALA, free $\mathrm{Na}^{131}$ I and PBS were added to the medium with different concentrations, and incubated for another 24 hours. After incubation, the CCK-8 assay was performed to determine the cell viability.

\section{Cellular uptake and localization of ${ }^{131}$ I-EM@ALA}

$4 \mathrm{~T} 1$ cells were seeded in 24-well plates at a density of $1 \times 10^{5}$ cells/well and cultured for 24 hours. Then ${ }^{131}$ I-EM@ALA ( $2 \mu \mathrm{ci} /$ well) or free $\mathrm{Na}^{131} \mathrm{I}(2 \mu \mathrm{ci} /$ well $)$ were added to the medium respectively. After incubated for different time points (1,2, 4, and 6 hours), cells and supernatant were collected and measured with a well-type $\mathrm{y}$-counter (2470 Automatic Gamma Counter WIZARD, PerkinElmer, Norwalk CT, USA).

4T1 cells were seeded into each 24-well plate at a concentration of $6 \times 10^{4}$ cells per well and cultured for 24 hours. After being treated with cy5 labeled EM@ALA for different time points, the tumor cells were observed by using a fluorescence confocal microscope or analyzed by flow cytometry. 


\section{Cerenkov radiation with ${ }^{131}$ I Radioisotope}

Cerenkov radiation was detected using an IVIS system (PerkinElmer Inc.) under various conditions (PBS, ALA, PpIX, $\mathrm{Na}^{131}$ I, $\mathrm{Na}^{131}$ I+ALA, and $\mathrm{Na}^{131}$ I+PpIX, all the drugs were free condition). The images were acquired with different emission filters, and the excitation was blocked.

\section{Western blot}

4T1 cells were seeded in 6-well plates at a density of $3 \times 10^{6}$ cells/well and cultured for 24 hours. Then PBS, EM@ALA, ${ }^{131}$ I-EM, ${ }^{131}$ I-EM@ALA were added to the medium respectively and cultivated for 24 hours. Total protein was gained from 4T1 cells by RIPA buffer (Beyotime Biotechnology, China). The tumorbearing mice were injected PBS, EM@ALA, ${ }^{131}$ I-EM (0.2 mci), ${ }^{131}$ I-EM@ALA (0.2 mci) respectively. Total protein was gained from the tumor of mice by RIPA buffer (Beyotime Biotechnology, China). The protein concentrations of tumor cells were measured with BCA protein Assay Kit (Beyotime Biotechnology, China). Equal amounts of protein $(20 \mu \mathrm{g})$ were added into each lane of a 10\% SDS-PAGE (New Cell \& Molecular Biotech, China) and separated. Then transferred the protein onto PVDF membranes (Millipore, United States), and the membranes were blocked by Protein Free Rapid Blocking Buffer (EpiZyme, China) for 2 hours and incubated with primary antibodies at $4^{\circ} \mathrm{C}$ for 12 hours. The primary antibodies used are as followed: anti-Caspase 3 (1:1000, Abclonal, China), anti-y-H2AX (1:1000, Proteintech, United States), anti-KU70 (1:5000, Proteintech, United States), anti-RAD51 (1:1000, Proteintech, United States), and antiGAPDH (1:1000, Abclonal, China). After incubation with HRP-coupled secondary antibody (goat antirabbit or rabbit anti-mouse IgG, 1:10000, Boster, China), the membranes were treated with chemiluminescence (Solarbio, China) for 3 mins and visualized on a Visionwork system.

\section{Animal models}

All animal studies were conducted under the guidance and approved by the Institutional Animal Care and Use Committee of Tongji Medical College of Huazhong University of Science and Technology. BALB/c mice (female, 5-6 weeks old) were purchased from Weitong Lihua Laboratory Animal Center (Beijing, China), and maintained in a pathogen-free environment. 4T1 cells $\left(1 \times 10^{7}\right.$ in $100 \mu \mathrm{L}$ PBS $)$ were subcutaneously injected into the right front leg of the BALB/C mice (Weitong Lihua Laboratory Animal Center, Beijing, China). The tumor-bearing mice were used for the following experiments after the tumor volume reached approximately $80 \mathrm{~mm}^{3}$. The tumor volume was calculated with the formula: length $\times$ width $^{2} \times 0.5$.

\section{In vivo fluorescence imaging}

When the tumor volumes ranged between 80-100 mm³ ICG-EM@ALA was injected to the tumor-bearing mice. The in vivo fluorescence imaging was conducted at different time points after injection. Tumors and organs of interest were resected and imaged 24 and 48 hours later. 
To detect the fluorescence signal of PpIX in vivo, 4T1 tumor bearing mice were sacrificed, and the interested tissues (e.g., heart, liver, kidneys, muscle, and tumor) were harvested for the fluorescence detection of PpIX by animal fluorescence imaging in 24 hours after EM@ALA injection.

\section{SPECT/CT imaging and biodistribution experiment}

Single-Photon Emission Computed Tomography/Computed Tomography (SPECT/CT) imaging was conducted to observe the whole-body distribution of ${ }^{131} \mathrm{I}$-EM@ALA in 4T1 tumor-bearing mice using a human SPECT/CT device (NM670, GE, United States). 4 days before the SPECT/CT imaging experiment, all mice were fed with $1 \%$ potassium iodide solution. The mice of experimental group were injected with ${ }^{131}$ I-EM@ALA $(0.2 \mathrm{mCi}, 0.1 \mathrm{~mL})$ while the mice of control group were injected with $\mathrm{Na}^{131} \mathrm{I}(0.2 \mathrm{mCi}, 0.1$ $\mathrm{mL})$. The SPECT/CT images were acquired 24 hours after the drug injection.

For the biodistribution experiment, 4T1 tumor-bearing mice ( $\mathrm{n}=4$ in each group) were injected with ${ }^{131} \mathrm{I}-$ EM@ALA or $\mathrm{Na}^{131} \mathrm{I}(0.2 \mathrm{mCi}, 0.1 \mathrm{~mL})$ via tail vein, and sacrificed at 24 hours. The organs (e.g., blood, heart, liver, muscle, tumor) of interest were harvested and weighed. Radioactivity of tissue was quantified using a $y$-counter.

\section{Therapeutic effect in animals}

To explore the ${ }^{131}$ I-EM@ALA treatment effect, the mice were divided into 4 groups ( $n=8$ per group) randomly for various treatments when the tumor volumes reached $80 \mathrm{~mm}^{3}$ : (1) PBS; (2) EM@ALA; (3) ${ }^{131} \mathrm{I}$-EM; (4) ${ }^{131} \mathrm{I}$-EM@ALA. The dosages of ${ }^{131} \mathrm{I}$ and ALA in these studies were $25 \mu \mathrm{Ci} / \mathrm{g}$ and $30 \mu \mathrm{g} / \mathrm{g}$, respectively. Tumor volume was measured once every 2 days for 19 days. The survival rate was assessed by the Kaplan-Meier method. The end point events were defined as follow: tumor volumes $>1500 \mathrm{~mm}^{3}$; ulcerating tumor tissue; mortality; $>15 \%$ weight loss.

\section{In vivo toxicity evaluation}

Group division and drugs injection were as described above ( $\mathrm{n}=4$ per group). In vivo toxicity was assessed using body weight change, hematoxylin and eosin (H\&E) staining, hematology and blood biochemistry indices. The general state of the animals was observed daily, and the body weight of the mice was monitored every other day. At the end of the 19-day treatment, whole blood was collected and analyzed to determine aspartate aminotransferase (AST), alanine aminotransferase (ALT), blood urea nitrogen (BUN), creatinine (CRE), and alkaline phosphatase (ALP) using a biochemical analyzer (Chemray240, China). Meanwhile, the samples of blood and interested tissues (heart, lung, liver, kidney, spleen, muscle, and thyroid) were harvested for H\&E staining. Besides, since we are interested in the toxicity differences between ${ }^{131} \mathrm{I}$-EM@ALA group and ${ }^{131} \mathrm{I}$-EM@PpIX group, tumor-bearing mice were sacrificed after 7, 14 and 21 days from the nanoparticle application, and samples of blood and interested tissues were harvested for the further analysis.

\section{Histological analysis}


Interested organs and tumor tissues were taken out from mice and fixed with $4 \%$ paraformaldehyde, then embedded in paraffin after 19-day treatment. The sliced interested organs and tumor tissues were further performed for H\&E, terminal deoxynucleotidyl transferase-mediated dUTP-biotin nick end-labeling (TUNEL) and Ki67 staining.

\section{Statistical analysis}

All values were presented as the means \pm standard deviation (SD). The significance level was set as 0.05 , and the data were presented with $(*)$ for $p<0.05$, (**) for $p<0.01$, and $(* \star *)$ for $p<0.001$, respectively.

\section{Results}

\section{Fabrication and characterization of EM@ALA}

Results from the transmission electron microscopy (TEM) showed the spherical morphology of EM@ALA and EM nanoparticles (Fig. 1a). Dynamic light scattering (DLS) experiments demonstrate that the mean radius of EM@ALA and EM nanoparticle was $154.8 \pm 2.8 \mathrm{~nm}$ and $144.4 \pm 1.4 \mathrm{~nm}$, respectively (Fig. 1b). To determine whether functionalized membrane proteins were retained on the EM@ALA and EM, the protein contents of EM@ALA and EM were analyzed by western blot. The results revealed that EM@ALA and EM presented a similar protein profile with $4 \mathrm{~T} 1$ cell lysate (Fig. 1c). The membrane-specific markers in EM@ALA and EM such as positive antigen CD44 were well inherited from the 4T1 cells, while the cytosol marker (i.e., glyceraldehyde 3-phosphate dehydrogenase (GAPDH)) and the nuclear protein marker (i.e., histone H3) were almost undetected in the final EM@ALA and EM. Additionally, the hydrodynamic sizes and Zeta potential of EM@ALA and EM did not change significantly for 7 days (Fig. $1 d, e)$, which indicated excellent stability of the nanoplatform. The radiolabeling efficiencies of ${ }^{131} \mathrm{I}$ EM@ALA was about $96.65 \%$ in PBS (Fig. 1f). The radiochemical stabilities were $80.5 \% \pm 1.3 \%$ in PBS and $74.3 \% \pm 2.2 \%$ in FBS for up to 48 hours (Fig. $1 \mathrm{~g}, \mathrm{~h}$ ), which suggesting that the radiolabeled ${ }^{131} \mathrm{I}$-EM@ALA nanoplatform has excellent biological stability in terms of size and radiochemistry profile, and could be applied to in vivo biological systems. In further experiments (Fig. 1i), ALA, which could be transferred to PpIX in mitochondrion, was identified to be the optimal PS because PpIX had ideally matched absorbance wavelength $\left(\lambda_{\mathrm{abs}} \varangle 480 \mathrm{~nm}\right.$ ) with Cerenkov light (major in the ultraviolet and blue end of the visible spectrum, $\lambda \otimes 480 \mathrm{~nm})$.

\section{Cellular uptake and treatment effects of ${ }^{131}$ I-EM@ALA}

Cy5 labelled EM@ALA incubated with 4T1 cells for different times, the results were analyzed by confocal laser scanning microscope and flow cytometry. Confocal images revealed strong Cy5-red fluorescence signals in the cytoplasm at 1 hour, 2 hour, and 3 hours (Fig. 2a). Flow cytometric analysis (Fig. 2b and 2c) showed that the tumor cell binding increased slightly over time, and no statistical difference between 1 hour incubation and 2 hours incubation ( $p=0.13)$, or 2 hours incubation and 3 hours incubation $(p=$ $0.08)$, but statistic difference was observed between 1 hour incubation and 3 hours incubation $(p \otimes 0.05)$. To further determine uptake rate accurately, we performed cell uptake assay by radionuclide. As 
illustrated in Figure 2d, the uptake of ${ }^{131} \mathrm{I}$-EM@ALA by 4T1 cells increased gradually over time and reached a maximum at 6 hours, which was significantly higher than that of free ${ }^{131} I(p \otimes 0.001)$.

Then, we verified the ability of ALA-derived PpIX accumulation in cancer cells. 24 hours after intravenously injection, we could clearly detect red fluorescence signal from PpIX in 4T1 cells (Fig. 2e). Subsequently, we examined the transformation of ALA to PpIX in vivo. The PpIX fluorescence signals in tumors and major organs showed that ALA was successfully converted to PpIX mainly within tumors (by endogenous enzymes), and little PpIX accumulated in livers (Fig. 2f). Semi-quantitative analysis showed that PpIX fluorescence intensity in tumors was about 1.8-fold than that in livers (Fig. $2 \mathrm{~g}$ ). As illustrated in Fig. $2 \mathrm{~h}$, the relative viabilities of the $4 \mathrm{~T} 1$ cells had a negative correlation with the ALA concentration or ${ }^{131}$ I activity. In the absence of laser light, EM@ALA had no obvious cytotoxicity on the $4 \mathrm{~T} 1$ cells at both concentrations. At an ALA concentration of $700 \mu \mathrm{g} / \mathrm{mL},{ }^{131}$ I radioactivity of $1000 \mu \mathrm{Ci} / \mathrm{mL}$, the viability of the $4 \mathrm{~T} 1$ cells was $100 \% \pm 3 \%, 96.8 \% \pm 18.5 \%, 47.3 \% \pm 2.4 \%, 33.1 \% \pm 1.9 \%, 8.8 \% \pm 0.6 \%$ with the treatment of PBS, EM@ALA, free $\mathrm{Na}^{131}$ I, ${ }^{131}$-EM and ${ }^{131}$ I-EM@ALA, respectively. ${ }^{131}$ I-EM@ALA group showed the best antitumor effects compared to other groups, which demonstrated the cooperative effect of radiotherapy and CR-PDT. Additionally, ${ }^{131} \mathrm{I}$-EM showed a better treatment effect than free $\mathrm{Na}^{131} \mathrm{I}$, which may because of the higher cell uptake of ${ }^{131}$ I-EM.

\section{Cerenkov radiation imaging}

To detect the Cerenkov radiation and CRET, we used the small-animal in vivo imaging system (IVIS) to collect ${ }^{131}$ I emission without excitation light. As shown in Fig. $3 a$ and $3 b$, the solution containing PBS, ALA and PpIX did not emit fluorescence, while we could detect obvious fluorescence signal emitted from the solutions containing ${ }^{131} \mathrm{I},{ }^{131}$ I plus ALA, and ${ }^{131}$ I plus PpIX, and the signal in ${ }^{131}$ I plus PpIX solution was higher than that in ${ }^{131}$ I plus ALA solution $(p \otimes 0.05)$. In the solutions containing ${ }^{131} I$ and PpIX, the fluorescence signal increased when the concentration of ${ }^{131} \mathrm{I}$ increased (fig.3c and 3d), so did the solutions containing only ${ }^{131}$ I without PpIX (Fig. s3), while the fluorescence signal decreased when the concentration of PpIX increased (fig.3e and $3 f$ ). These results indicate that the CR energy could transfer to PpIX from CR.

\section{In vivo NIRF imaging}

The distribution of the nanoparticle in vivo was investigated by intravenously injecting ICG-EM@ALA to 4T1 tumor-bearing mice. The in vivo fluorescence signals in tumors gradually increased over time and reached maximum intensity at 24 hours, suggesting the time-dependent accumulation of ICG-labeled EM@ALA in the tumor (Fig. 4a). The T/M ratio peaked at 24 hours after injection (1.7 \pm 0.1 ; Fig. 4b). The ex vivo fluorescence images further confirmed the accumulation of ICG-EM@ALA in tumor site. Remarkably, strong fluorescence was also detected in the livers, likely contributed to the clearance of small nanoparticles EM@ALA by livers (Fig. 4c and 4d). 
To further confirm the accumulation of ${ }^{131} \mathrm{I}$-EM@ALA in tumor sites, SPECT/CT imaging was carried out to evaluate the distribution of ${ }^{131} \mathrm{I}$-EM@ALA in vivo. With reference to the fluorescence imaging results, SPECT/CT images were gained at 24 hours after-injection. The signal in the tumor region was greatly higher than that in surrounding tissues (Fig. s4). The bio-distribution results showed that the T/B (tumor to blood) and T/M (tumor to muscle) were about $2.1 \pm 0.6$ and $4.7 \pm 1.1$, respectively (Fig. s4). The results of imaging and biodistribution showed that ${ }^{131}$ I-EM@ALA could effectively accumulated in tumor region.

\section{In vivo tumor therapy in $4 \mathrm{~T} 1$ tumor-bearing mice model}

We then examined whether the combination CR-PDT and radiotherapy could effectively inhibit tumor growth. At 19 days after treatment, the tumor volumes of the ${ }^{131}$ I-EM@ALA group $\left(580.0 \pm 146.4 \mathrm{~mm}^{3}\right)$ were significantly smaller than those from the ${ }^{131}$ I-EM $\left(1081.5 \pm 273.5 \mathrm{~mm}^{3}\right)$ or EM@ALA groups $\left(1414.9 \pm 194.9 \mathrm{~mm}^{3}\right)$, which showed that combination therapy had greater antitumor efficacy than single ${ }^{131}$ I-radiotherapy (Fig. 5a). Notably, there were no statistical differences between EM@ALA group and control group, indicating the ${ }^{131}$ I CR was critically important in CR-PDT. For this experiment, the survival rates were recorded for 35 days (Fig. 5b). The ${ }^{131}$ I-EM@ALA group (12.5\%, 35 days) and ${ }^{131}$ IEM@PpIX group $(25 \%, 35$ days) greatly prolonged the survival time compared to the control group $(0 \%, 24$ days). At the end of the treatments, the tumors were harvested (Fig. 5c) and stained (Fig. 5d). HE staining of the tumor tissues showed decreased nucleus-to-cytoplasm ratios, which were related to the decreased number of cancer cells in mice injected with ${ }^{131}$ I-EM@ALA. Ki67 staining and TUNEL staining showed that treatment with ${ }^{131}$ I-EM@ALA and ${ }^{131}$ I-EM@PpIX induced the highest rate of apoptosis and an optimal level of necrotic lesions.

\section{In vivo toxicity evaluation}

There was no obvious weight loss in all groups during the 19-days experiment (Fig. 6a). The results showed that both routine blood parameters and blood biochemistry parameters were within the normal range of fluctuations (Fig. 6b, 6c and 6d). Representative HE staining showed there were no obvious histological damage (Fig. 6e). These results suggest the excellent biocompatibility of ${ }^{131}$ I-EM@ALA.

We compared the side effect of ${ }^{131} \mathrm{I}$-EM@ALA and ${ }^{131} \mathrm{I}$-EM@PpIX in vivo to evaluate the effect of reducing potential toxicity of our strategy. As in shown in Fig. 7a-c, the RBC, PLT, and HGB in ${ }^{131}$ I-EM@PpIX group were lower than that of ${ }^{131}$ I-EM@ALA group both in 7,14 and 21 days $(p \otimes 0.05)$ except PLT in 14 days ( $p$ $=0.18$ ), indicting impaired blood cells. At 21 days after treatments, the levels of AST, ALT, and ALP in ${ }^{131}$ IEM@PpIX group were significantly higher than that in ${ }^{131}$ I-EM@ALA group (Fig. 7d), indicting hepatocytes were damaged in ${ }^{131} \mathrm{I}$-EM@PpIX. In HE staining, severe oedema of hepatocytes was observed in ${ }^{131}$ IEM@PpIX group but not in ${ }^{131}$ I-EM@ALA group in 7 days, 14 days, and 21 days (Fig. 7e), indicating terrible damages in liver cells in ${ }^{131}$ I-EM@PpIX group, and we inferred that the damages were caused by CR-PDT but not radiotherapy. All the results showed that our strategy of using photosensitizer precursors ALA instead of photosensitizers (such as PpIX) could greatly reduce the adverse effects. 


\section{The mechanism of tumor cell apoptosis}

Most recent studies have shown that PDT leads to tumor death via the lysosomal pathway $(27,28)$. The in vitro expression of apoptosis related proteins (caspase 3 ) and radiological DNA damage and repair related proteins ( $\mathrm{Y}-\mathrm{H} 2 \mathrm{AX}, \mathrm{KU70}$, and RAD51)(29-32) was investigated in Fig. 8a and 8b. The expression of caspase 3 in ${ }^{131}$ I-EM@ALA group was greatly higher than that in ${ }^{131}$ I-EM group $(P \otimes 0.01)$ and control group ( $P \otimes 0.001)$. Similarly, the expression of radiological DNA damage and repair related proteins (KU70, and RAD51) in ${ }^{131}$ I-EM@ALA group was higher than that in ${ }^{131}$ I-EM group (P $\left.\otimes 0.001\right)$ and control group $(\mathrm{P} \otimes 0.001)$. As for the in vivo experiment, the expression of KU70 in ${ }^{131} \mathrm{I}$-EM@ALA group was lower than that of ${ }^{131}$ I-EM@group (Fig. 8c and 8d).

\section{Discussion}

In this study, we designed an exosome-mimetic nanoparticles loading ALA and modified with radionuclide ${ }^{131}$ I ( ${ }^{131}$ I-EM@ALA), which were proved to be stable in structure, excellent in anti-tumor effects, and had no obvious side effects. The strategy of using CR to excite photosensitizer PpIX which was converted from ALA had effectively suppressed the tumor growth and minimized the side effects to normal organs especially liver and blood cells, which is due to the conversion of ALA to PpIX mainly occurred in tumor sites but rarely in normal tissues. Our study offers a new therapeutic avenue for PDT that does not rely on external light sources and minimized the side effects.

Biomimetic nanoparticles encapsulated with an active cell membrane are attracting increasing attention(33,34). Owing to the reserved cell membrane structure, biomimetic nanoparticles can acquire special functions, such as long blood circulation, and immune escaping, powerful drug delivery vehicle, and vaccination $(35,36)$. Cancer cells can realize the intercellular homologous junctions with membrane proteins because of the surface adhesion molecules expressed on cancer cells. Cancer cell membranecoated nanoparticles, therefore, are expected to obtain homologous targeting ability, which is regarded as the ideal vehicle for drug delivery and effective cancer therapy. Our study reached the same conclusion. ${ }^{131}$ I-EM@ALA could effectively target to the tumor cells both in vitro and in vivo (Fig. 2a, and 4a). Besides, the results of DLS, TEM, and WB proved that the properties of exosome-mimetics nanoparticles did not change significantly after modification. The radiolabeling stability was $80.5 \% \pm 1.3 \%$ in PBS and $70.4 \% \pm 2.2 \%$ in FBS in 48 hours, which is good enough for the subsequent studies. In the CR imaging and CRET imaging experiments, we found that our designed CR-PDT strategy has an obvious dosedependent effect (Fig. 3a-f), and the results demonstrated that PpIX could be excited by the UV/blue CL of radionuclide ${ }^{131} \mathrm{I}$, which then in turn induced PDT.

We next verified the synergistic therapeutic effects of radiotherapy and CR-PDT in the 4T1 tumor-bearing mice. Cell viability was evaluated and showed that ${ }^{131}$ I-EM@ALA group had the best antitumor effect than the groups of ${ }^{131} \mathrm{I}$-EM or EM@ALA, showing that both ${ }^{131} \mathrm{I}$ and ALA are indispensable in CR-PDT. In the further animal experiments, the combined radio-photodynamic therapy in ${ }^{131} \mathrm{I}$-EM@ALA group 
markedly inhibited tumor growth and prolonged the survival time. The results of HE staining, Ki67 staining, and TUNEL staining showed the highest rate of apoptosis of ${ }^{131} \mathrm{I}$-EM@ALA. It is worth noting that in our CR-PDT antitumor study, substitution of photosensitizer PpIX with photosensitizer precursor ALA did not significantly reduce the efficacy of antitumor therapy.

Subsequently, we assessed the side effect of combined radio-photodynamic therapy. The potential combination strategy by coupling radionuclide with photosensitizer might continuously generate ROS during its blood circulation which make this modality effectively a normal chemotherapeutic agent. The spatiotemporal colocalization of the photosensitizer with the radionuclide in these studies resulted in potential side effects to healthy tissues(12). In this study, we designed a nanoparticle which loading ALA and labeled with ${ }^{131} \mathrm{I}$; in tumor site, ALA is converted to PpIX in a large number of mitochondria, and CRPDT is activated in the presence of photosensitizer PpIX and ${ }^{131} \mathrm{I}$-derived Cerenkov radiation and leading to tumor death. While in normal tissues, ALA is rarely converted to PpIX and PDT could not be activated, leading to limited side effect to normal tissues. After the 19-day treatment, there was no observable weight loss, and routine blood parameters, blood biochemistry parameters, and HE staining were within the normal range of fluctuations in ${ }^{131} \mathrm{I}$-EM@ALA group, but liver damage could be clearly observed in ${ }^{131}$ I-EM@PpIX group (Fig. 6a-e). Therefore, we next compared the differences of side effects between ${ }^{131}$ IEM@ALA and ${ }^{131}$ I-EM@PpIX. We monitored the levels of RBC, HGB, and PLT and liver HE staining every week. The results showed that ${ }^{131} \mathrm{I}$-EM@PpIX caused severe blood cells damage and hepatocyte damage, but these injuries did not occur in the ${ }^{131}$ I-EM@ALA group (Fig. 7a-e). The possible reason maybe that ${ }^{131}$ I-EM@PpIX continuously produced ROS during its blood circulation especially in blood and liver (the EM nanoparticles would accumulate in liver, Fig. 4a).

\section{Conclusion}

In summary, a multifunctional platform, ${ }^{131}$ I-EM@ALA, was successfully synthesized for effective delivery of ${ }^{131} \mathrm{I}$ and ALA. Based on the changed blood circulation, ${ }^{131} \mathrm{I}$-EM@ALA improved ${ }^{131} \mathrm{I}$ and ALA delivery into cancer cells both in vitro and in vivo, thereby promoting enhanced therapy effect. ${ }^{131}$ I not only plays a role in radiotherapy, but its $\mathrm{CR}$ can act as an internal light source. The results showed that one-third of subcutaneous tumor-bearing mice could be eradicated by only one injection of ${ }^{131}$ I-EM@ALA. By comparing the potential toxicity difference between ${ }^{131}$ I-EM@ALA group and ${ }^{131}$ I-EM@PpIX group, we confirmed that our strategy of using ALA which was a photosensitizer precursor could effectively reduce the side effects. Our study provides a novel multi-therapeutic concept for antitumor study, as well as gives a new vision for combined therapeutic effects by synergistic radiotherapy and CR-PDT without external light source.

\section{Abbreviations}

PDT: Photodynamic therapy; CR: Cerenkov radiation; CR-PDT: the Cerenkov radiation-induced PDT; ALA: 5-Aminolevulinic acid; ROS: Reactive oxygen species; EM: Exosome-mimetic; PpIX: Protoporphyrin IX; 
PSs: Photosensitizers; EPR: the enhanced permeability and retention; RT: radiation therapy; FBS: fetal bovine serum; TEM: transmission electron microscope; WB: western blot; iTLC-SG: The instant thin-layer chromatography-silica gel; SPECT/CT: Single-Photon Emission Computed Tomography/Computed Tomography; AST: aspartate aminotransferase; ALT: alanine aminotransferase; BUN: blood urea nitrogen; CRE: creatinine; ALP: alkaline phosphatase; WBC: White blood cells; Neu\%: neutrophil percentage; Lymph\%: lymphocytes percentage; Mon\%: monocyte percentage; RBC: red blood cells; HCT: hematocrit; HGB: hemoglobin; $\mathrm{MCH}$ : mean corpuscular hemoglobin; MCV: mean corpuscular volume; MCHC: mean corpuscular hemoglobin concentration; PLT: Platelets; MPV: mean platelet volume; RDW: red cell volume distribution width H\&E staining: hematoxylin and eosin staining; TUNEL staining: terminal deoxynucleotidyltransferase-mediated dUTP-biotin nick end-labeling staining; SD: standard deviation; TEM: the transmission electron microscopy; DLS: Dynamic light scattering; GAPDH: glyceraldehyde 3-phosphate dehydrogenase;

\section{Declarations}

\section{Contributor Information}

Rui An, Email: 1975xh0577@hust.edu.cn

Zairong Gao, Email: gaobonn@163.com

\section{Footnotes}

Ruijie Qian and Kun Wang have contributed equally to this work.

\section{Ethics approval and consent to participate}

All experimental schemes were performed under the guidance and approved by the Institutional Animal Care and Use Committee of Tongji Medical College of Huazhong University of Science and Technology. Balb/c mice (female, 5-6 weeks old) purchased from Weitong Lihua Laboratory Animal Center (Beijing, China), and maintained in a pathogen-free environment. Extensive efforts were made to ensure minimal suffering of the animals used during the study.

\section{Consent for publication}

All authors agree to be published.

\section{Availability of data and materials}

The datasets used and analyzed during the current study are available from the corresponding author on reasonable request.

\section{Competing interests}


The authors have declared that no competing interest exists.

\section{Funding}

This work was supported by the National Natural Science Foundation of China (No. 82071966 and 81771866).

\section{Authors' contributions}

ZRG and RA contributed to the design and supervision of this study. RJQ and KW performed the experiments. YWG and ZYZ analyzed the data and interpretation. $L X L, D W J, H Y L, X J H, C P G$ and YG provided suggestions and technical support for this study. RJQ, KW, RG and CYW wrote the manuscript. All authors read and approved the final manuscript.

\section{Acknowledgements}

This work was supported by the National Natural Science Foundation of China (No. 82071966 and 81771866).

We would like to thank Miss Guang-Xin Wang and Miss Yan Wang at The Analysis and Testing Center of Institute of Hydrobiology, Chinese Academy of Sciences for discussion and comments on the manuscript.

\section{References}

1. P. Agostinis et al., Photodynamic therapy of cancer: an update. CA: a cancer journal for clinicians 61, 250-281 (2011).10.3322/caac.20114.

2. V. S. Chelakkot et al., MEK reduces cancer-specific PpIX accumulation through the RSK-ABCB1 and HIF-1a-FECH axes. Scientific reports 10, 22124-22124 (2020).10.1038/s41598-020-79144-x.

3. D. Ni et al., Magnetic Targeting of Nanotheranostics Enhances Cerenkov Radiation-Induced Photodynamic Therapy. Journal of the American Chemical Society 140, 14971-14979 (2018).10.1021/jacs.8b09374.

4. S. Goel et al., Activatable Hybrid Nanotheranostics for Tetramodal Imaging and Synergistic Photothermal/Photodynamic Therapy. Advanced materials (Deerfield Beach, Fla.) 30, (2018).10.1002/adma.201704367.

5. N. Kotagiri, G. P. Sudlow, W. J. Akers, S. Achilefu, Breaking the depth dependency of phototherapy with Cerenkov radiation and low-radiance-responsive nanophotosensitizers. Nature nanotechnology 10, 370-379 (2015).10.1038/nnano.2015.17.

6. X. Ai et al., In vivo covalent cross-linking of photon-converted rare-earth nanostructures for tumour localization and theranostics. Nature communications 7, 10432 (2016).10.1038/ncomms10432. 
7. C. Zhang et al., Marriage of scintillator and semiconductor for synchronous radiotherapy and deep photodynamic therapy with diminished oxygen dependence. Angewandte Chemie (International ed. in English) 54, 1770-1774 (2015).10.1002/anie.201408472.

8. D. Duan et al., Activating TiO(2) Nanoparticles: Gallium-68 Serves as a High-Yield Photon Emitter for Cerenkov-Induced Photodynamic Therapy. ACS applied materials \& interfaces 10, 5278-5286 (2018).10.1021/acsami.7b17902.

9. A. Kamkaew et al., Cerenkov Radiation Induced Photodynamic Therapy Using Chlorin e6-Loaded Hollow Mesoporous Silica Nanoparticles. ACS applied materials \& interfaces 8, 26630-26637 (2016).10.1021/acsami.6b10255.

10. T. M. Shaffer, E. C. Pratt, J. Grimm, Utilizing the power of Cerenkov light with nanotechnology. Nature nanotechnology 12, 106-117 (2017).10.1038/nnano.2016.301.

11. P. Cai et al., A chlorin-lipid nanovesicle nucleus drug for amplified therapeutic effects of lung cancer by internal radiotherapy combined with the Cerenkov radiation-induced photodynamic therapy. Biomater Sci 8, 4841-4851 (2020).10.1039/d0bm00778a.

12. B. Yu et al., A "Missile-Detonation" Strategy to Precisely Supply and Efficiently Amplify Cerenkov Radiation Energy for Cancer Theranostics. Advanced materials (Deerfield Beach, Fla.) 31, e1904894 (2019).10.1002/adma.201904894.

13. Y. Xia et al., Engineering Macrophages for Cancer Immunotherapy and Drug Delivery. Advanced materials (Deerfield Beach, Fla.) 32, e2002054 (2020).10.1002/adma.202002054.

14. J. G. van den Boorn, J. Dassler, C. Coch, M. Schlee, G. Hartmann, Exosomes as nucleic acid nanocarriers. Advanced drug delivery reviews 65, 331-335 (2013).10.1016/j.addr.2012.06.011.

15. S. M. van Dommelen et al., Microvesicles and exosomes: opportunities for cell-derived membrane vesicles in drug delivery. Journal of controlled release : official journal of the Controlled Release Society 161, 635-644 (2012).10.1016/j.jconrel.2011.11.021.

16. T. R. Lunavat et al., RNAi delivery by exosome-mimetic nanovesicles - Implications for targeting cMyc in cancer. Biomaterials 102, 231-238 (2016).10.1016/j.biomaterials.2016.06.024.

17. R. Molinaro et al., Biomimetic proteolipid vesicles for targeting inflamed tissues. Nat Mater 15, 10371046 (2016).10.1038/nmat4644.

18. S. Tan, T. Wu, D. Zhang, Z. Zhang, Cell or cell membrane-based drug delivery systems. Theranostics 5, 863-881 (2015).10.7150/thno.11852.

19. Y. W. Choo et al., M1 Macrophage-Derived Nanovesicles Potentiate the Anticancer Efficacy of Immune Checkpoint Inhibitors. ACS Nano 12, 8977-8993 (2018).10.1021/acsnano.8b02446. 
20. H. Fang et al., Biomimetic oxygen delivery nanoparticles for enhancing photodynamic therapy in triple-negative breast cancer. Journal of nanobiotechnology 19, 81 (2021).10.1186/s12951-021-00827-2.

21. M. Li et al., Red blood cell membrane-coated upconversion nanoparticles for pretargeted multimodality imaging of triple-negative breast cancer. Biomater Sci 8, 1802-1814 (2020).10.1039/d0bm00029a.

22. H. Fang et al., Ultra-sensitive Nanoprobe Modified with Tumor Cell Membrane for UCL/MRI/PET Multimodality Precise Imaging of Triple-Negative Breast Cancer. Nanomicro Lett 12, 62 (2020).10.1007/s40820-020-0396-4.

23. X. Xu et al., Broaden sources and reduce expenditure: Tumor-specific transformable oxidative stress nanoamplifier enabling economized photodynamic therapy for reinforced oxidation therapy. Theranostics 10, $10513-10530$ (2020).10.7150/thno.49731.

24. H. Cheng et al., Mitochondria and plasma membrane dual-targeted chimeric peptide for single-agent synergistic photodynamic therapy. Biomaterials 188, 1-11 (2019).10.1016/j.biomaterials.2018.10.005.

25. S. Y. Li et al., Cancer cell membrane-coated biomimetic platform for tumor targeted photodynamic therapy and hypoxia-amplified bioreductive therapy. Biomaterials 142, 149-161 (2017).10.1016/j.biomaterials.2017.07.026.

26. J. A. Mol, T. J. Visser, Synthesis and some properties of sulfate esters and sulfamates of iodothyronines. Endocrinology 117, 1-7 (1985).10.1210/endo-117-1-1.

27. S. Qi et al., Hypocrellin A-based photodynamic action induces apoptosis in A549 cells through ROSmediated mitochondrial signaling pathway. Acta Pharm $\operatorname{Sin}$ B 9, 279-293 (2019).10.1016/j.apsb.2018.12.004.

28. W. K. Martins et al., Parallel damage in mitochondria and lysosomes is an efficient way to photoinduce cell death. Autophagy 15, 259-279 (2019).10.1080/15548627.2018.1515609.

29. U. Eberlein et al., DNA Damage in Peripheral Blood Lymphocytes of Thyroid Cancer Patients After Radioiodine Therapy. J Nucl Med 57, 173-179 (2016).10.2967/jnumed.115.164814.

30. J. Zhang et al., gamma-H2AX responds to DNA damage induced by long-term exposure to combined low-dose-rate neutron and gamma-ray radiation. Mutat Res Genet Toxicol Environ Mutagen 795, 36-40 (2016).10.1016/j.mrgentox.2015.11.004.

31. A. G. McCluskey et al., Inhibition of poly(ADP-Ribose) polymerase enhances the toxicity of 131Imetaiodobenzylguanidine/topotecan combination therapy to cells and xenografts that express the noradrenaline transporter. J Nucl Med 53, 1146-1154 (2012).10.2967/jnumed.111.095943. 
32. M. Gachechiladze, J. Skarda, A. Soltermann, M. Joerger, RAD51 as a potential surrogate marker for DNA repair capacity in solid malignancies. Int J Cancer 141, 1286-1294 (2017).10.1002/ijc.30764.

33. Z. Tang, P. Zhao, H. Wang, Y. Liu, W. Bu, Biomedicine Meets Fenton Chemistry. Chemical reviews 121, 1981-2019 (2021).10.1021/acs.chemrev.0c00977.

34. M. Izci, C. Maksoudian, B. B. Manshian, S. J. Soenen, The Use of Alternative Strategies for Enhanced Nanoparticle Delivery to Solid Tumors. Chemical reviews 121, 1746-1803 (2021).10.1021/acs.chemrev.0c00779.

35. H. Ding et al., Erythrocyte membrane-coated NIR-triggered biomimetic nanovectors with programmed delivery for photodynamic therapy of cancer. Nanoscale 7, 9806-9815 (2015).10.1039/c5nr02470f.

36. C.-M. J. Hu, R. H. Fang, B. T. Luk, L. Zhang, Nanoparticle-detained toxins for safe and effective vaccination. Nature nanotechnology 8, 933-938 (2013).10.1038/nnano.2013.254.

\section{Figures}

a

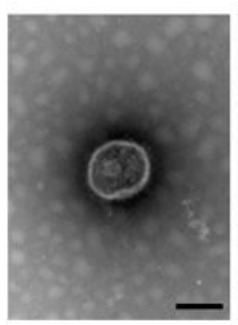

EM

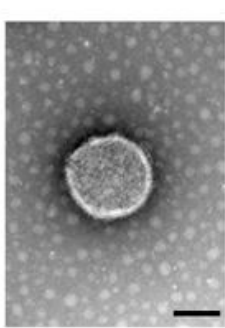

EM@ALA

d

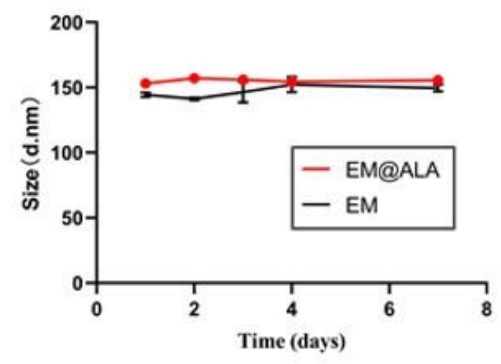

g

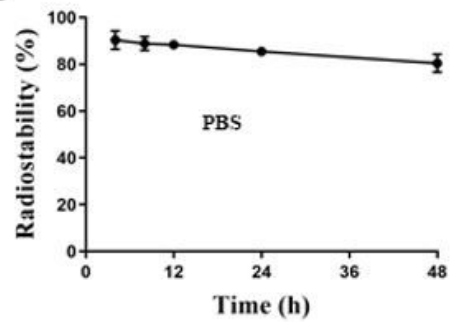

b

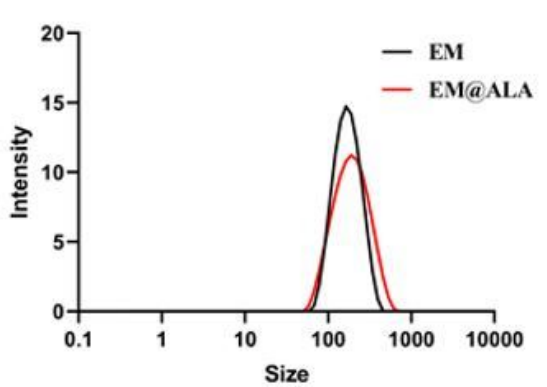

e

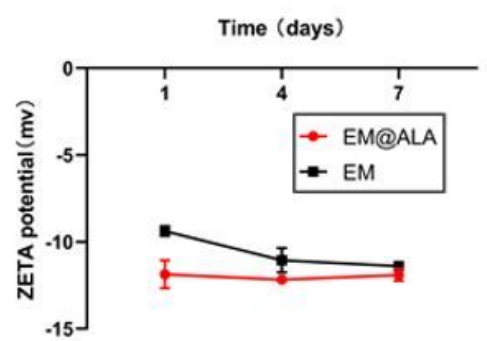

h

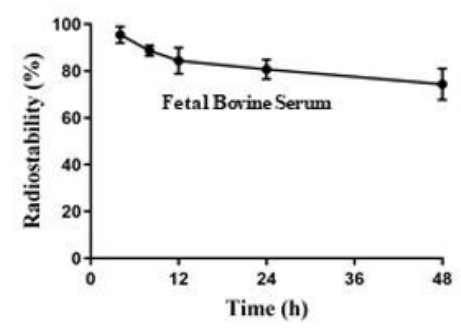

c $\quad$ CD44

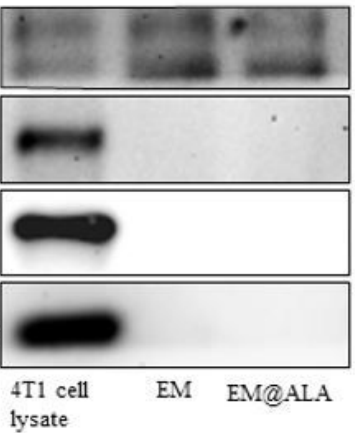

f

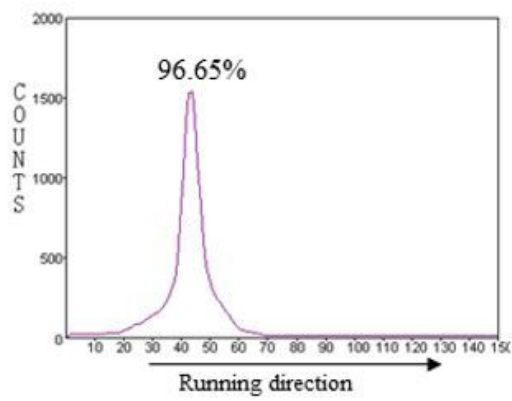

i

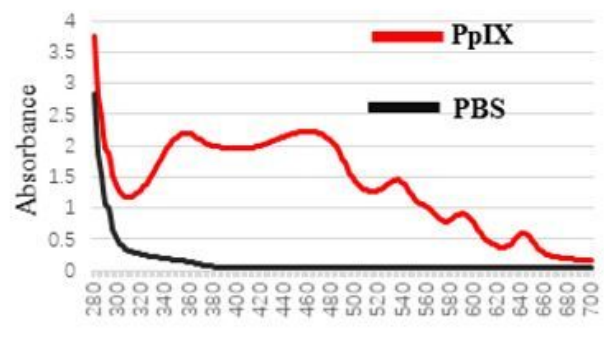


Figure 1

Characteristics of ${ }^{131}$ I-EM@ALA. 4T1 tumor cells derived drug-loaded EM and empty EM assessed by TEM (a), DLS (b) and WB (c). Scale bars $=100 \mathrm{~nm}$. Size(d) and Zeta potential(e) stability test of EM@ALA and EM in PBS for 7 days. (f) The representative radiochemical purity of ${ }^{131}$ I-EM@ALA. Radiolabeling stability test in PBS $(\mathbf{g})$ and FBS (h) during 48 hours $(n=3)$. (i) UV-vis spectrum of PpIX and PBS.
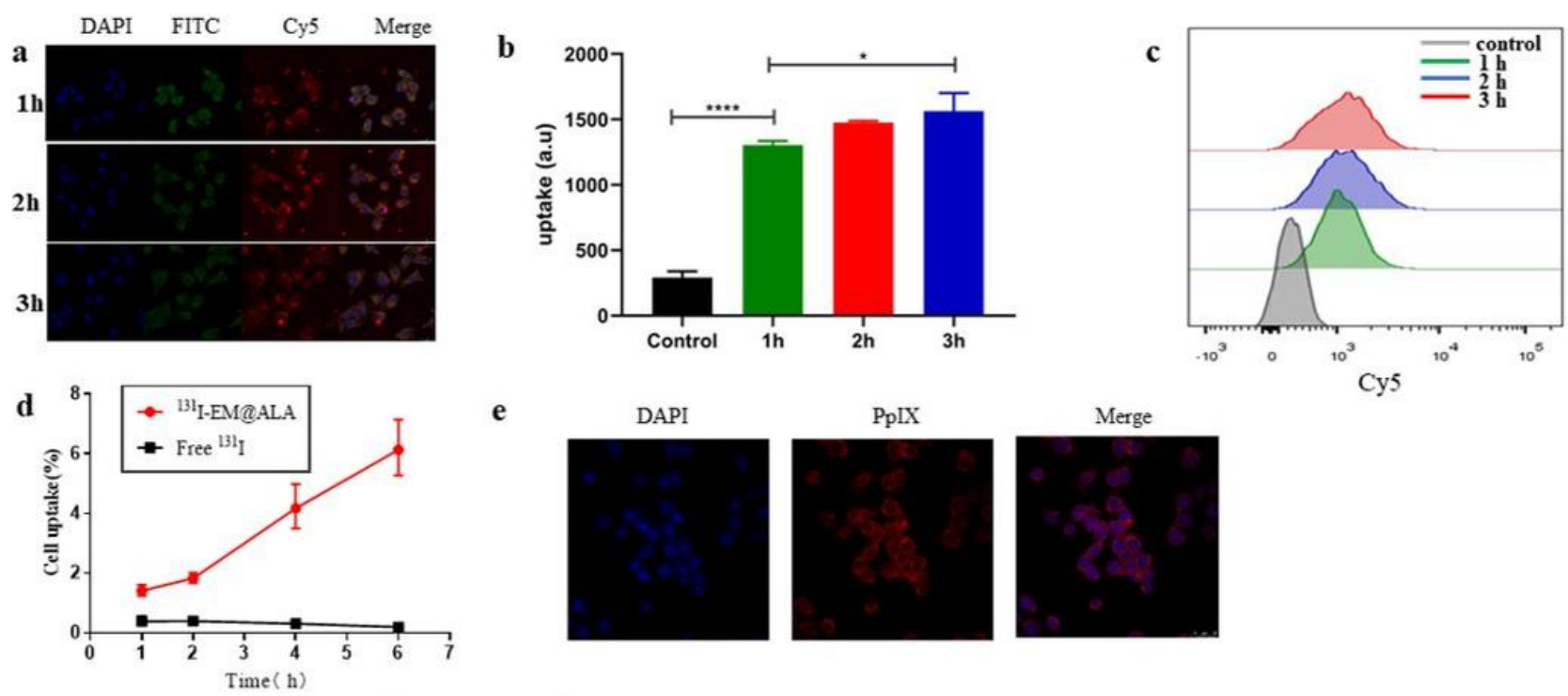

e

DAPI

PpIX
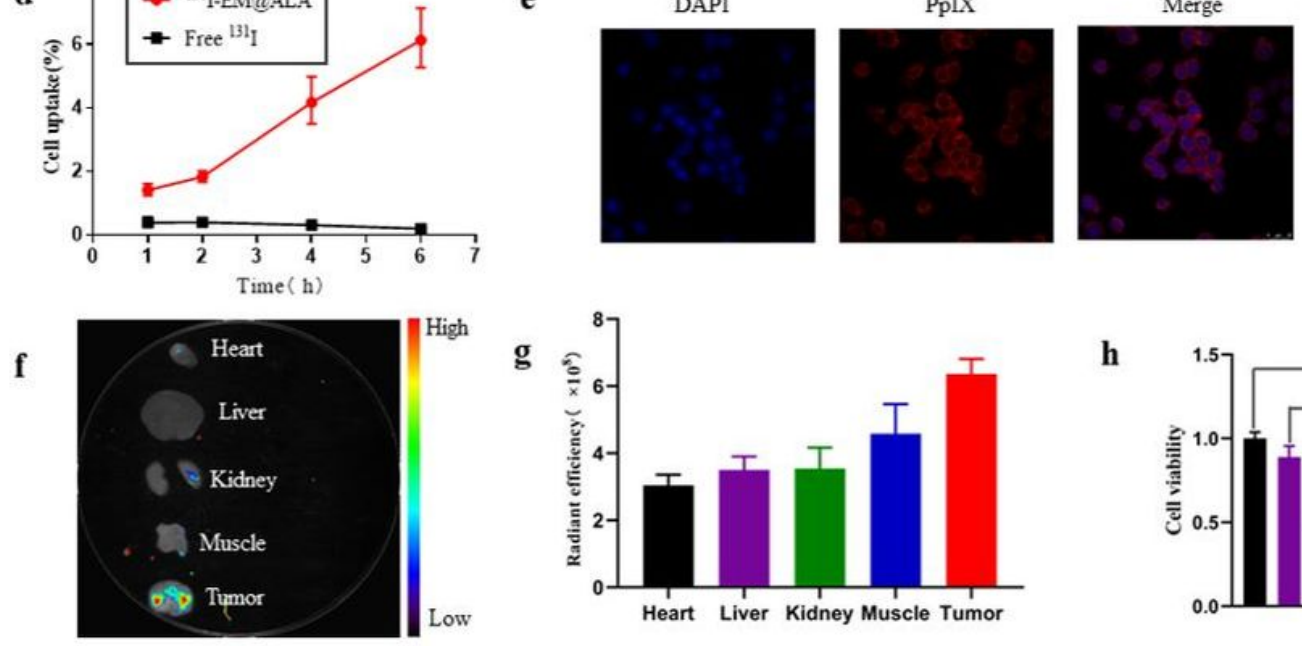

h

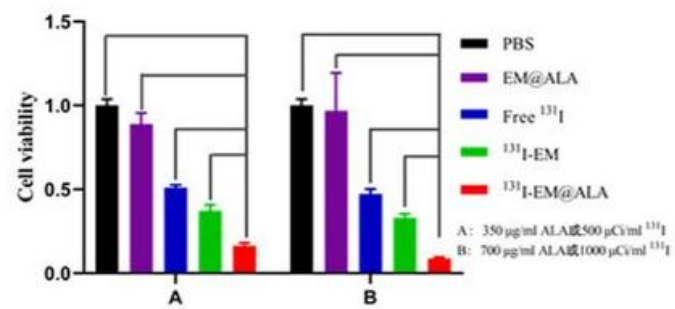

Figure 2

Tumor-binding and antitumor effects of ${ }^{131}$ I-EM@ALA. Confocal fluorescence images (a) and flow cytometry ( $\mathbf{b}$ and $\mathbf{c}$ ) of tumor cells after incubation with Cy5@EM@ALA for different times. Cell nuclei were stained blue with DAPI, filamentous actin cytoskeletons were stained green with FITC phalloidin. (d) In vitro uptake of ${ }^{131}$ I labeled EM@ALA or free $\mathrm{Na}^{131}$ I by $4 \mathrm{~T} 1$ cells at different points. (e) EM@ALA and 4T1 cells were incubated for 2 hours before visualization of PpIX-specific fluorescence by confocal microscopy. 24 hours after EM@ALA injection, ex vivo fluorescence images (f) of PpIX signal and semiquantitative analysis $(\mathbf{g})$. (h) Viability of $4 \mathrm{~T} 1$ cells with various treatments. 
a

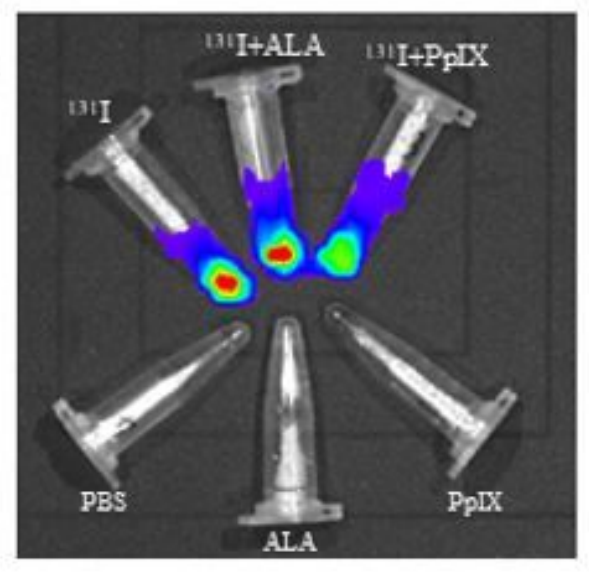

c

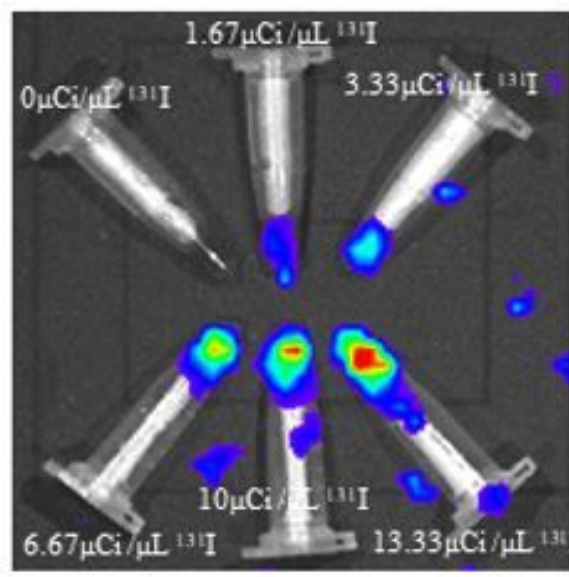

e

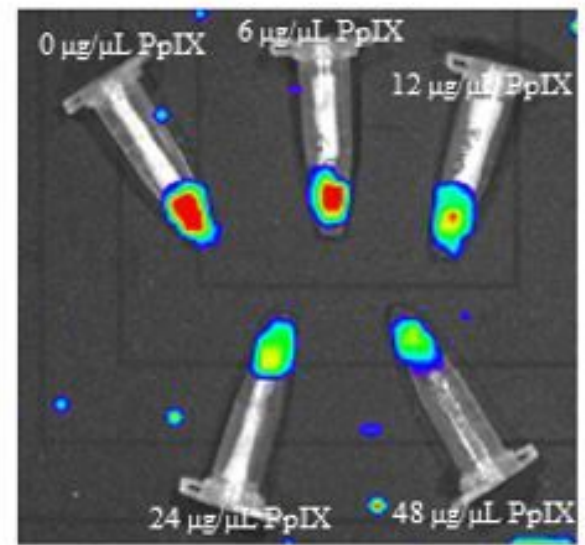

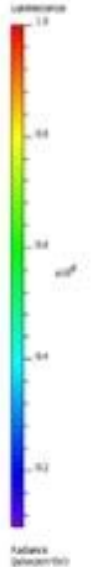

b

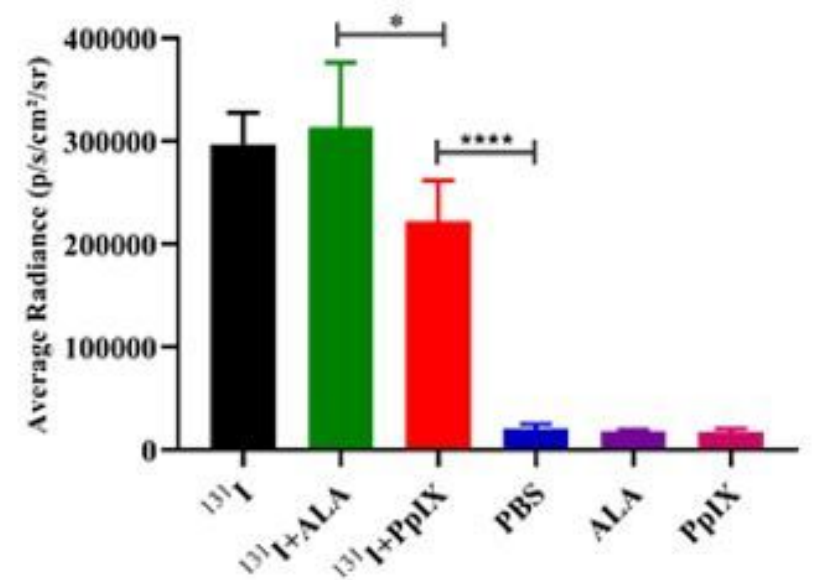

d

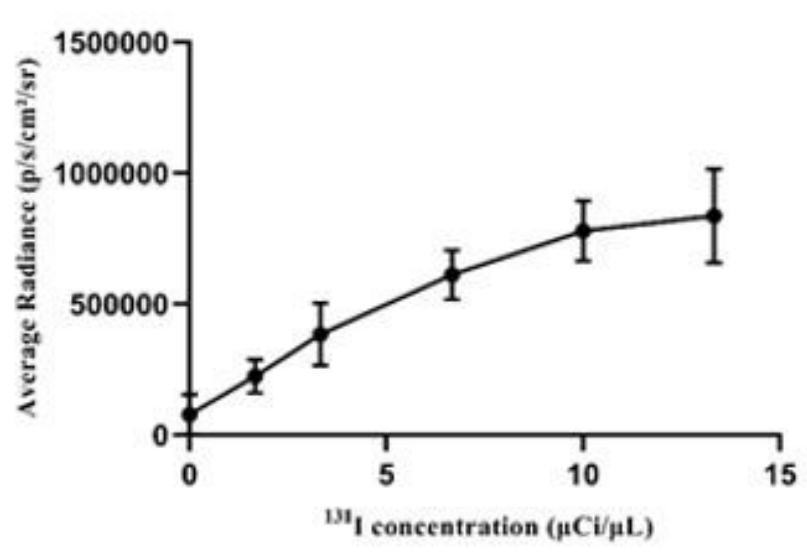

f

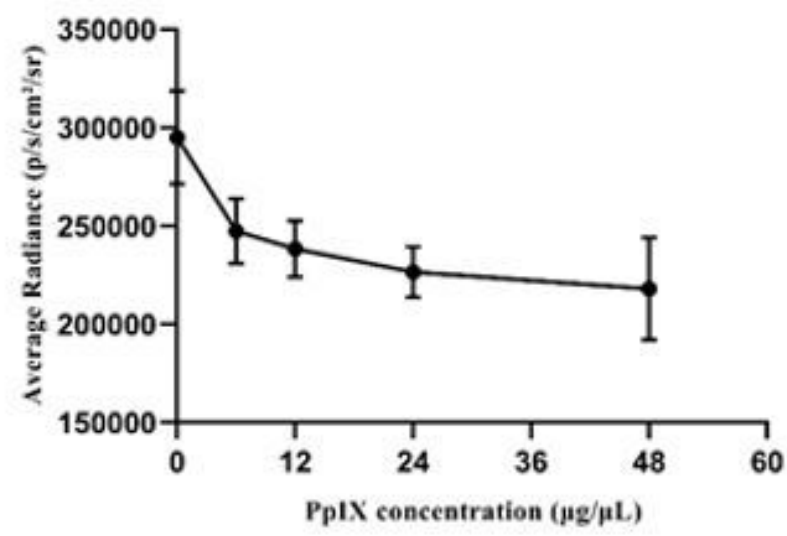

\section{Figure 3}

Cerenkov radiation. (a) Cerenkov radiation imaging in different kinds of solutions and the semiquantitative analysis (b). (c) Cerenkov radiation imaging with $1.5 \mu \mathrm{g} / \mu \mathrm{L}$ PpIX and different concentration of ${ }^{131}$ I and the semi-quantitative analysis (d). (e) Cerenkov radiation imaging with $4 \mu \mathrm{Ci} / \mu \mathrm{L}{ }^{131} \mathrm{I}$ and different concentration of PpIX and the semi-quantitative analysis (f). 
a

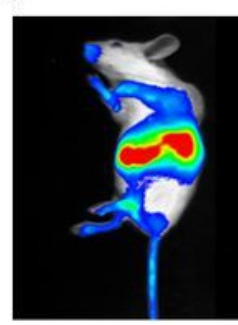

$1 \mathrm{~h}$

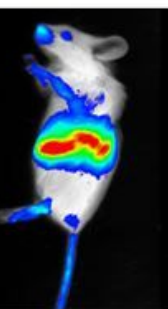

2h

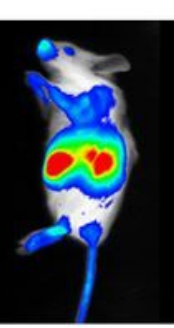

$5 \mathrm{~h}$

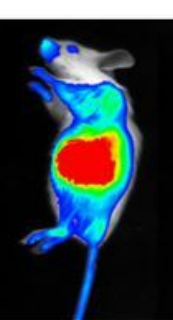

$10 \mathrm{~h}$

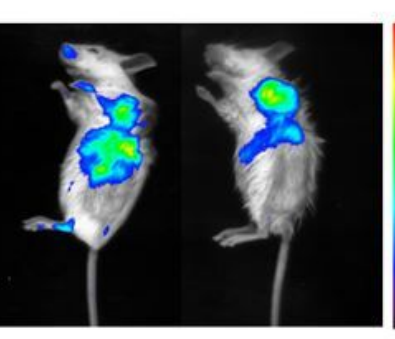

24h

$48 h$

c

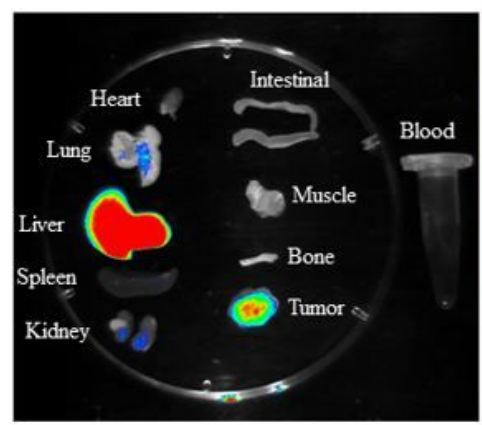

24h

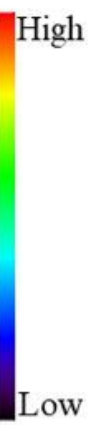

Low

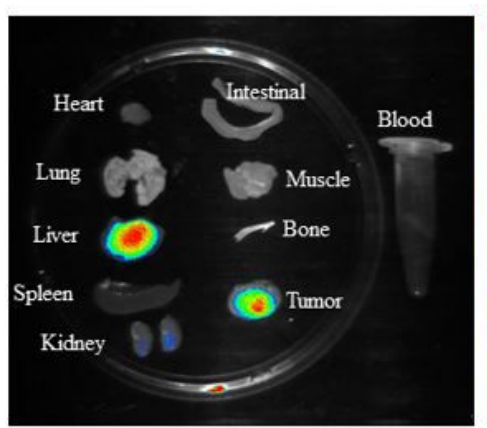

$48 \mathrm{~h}$
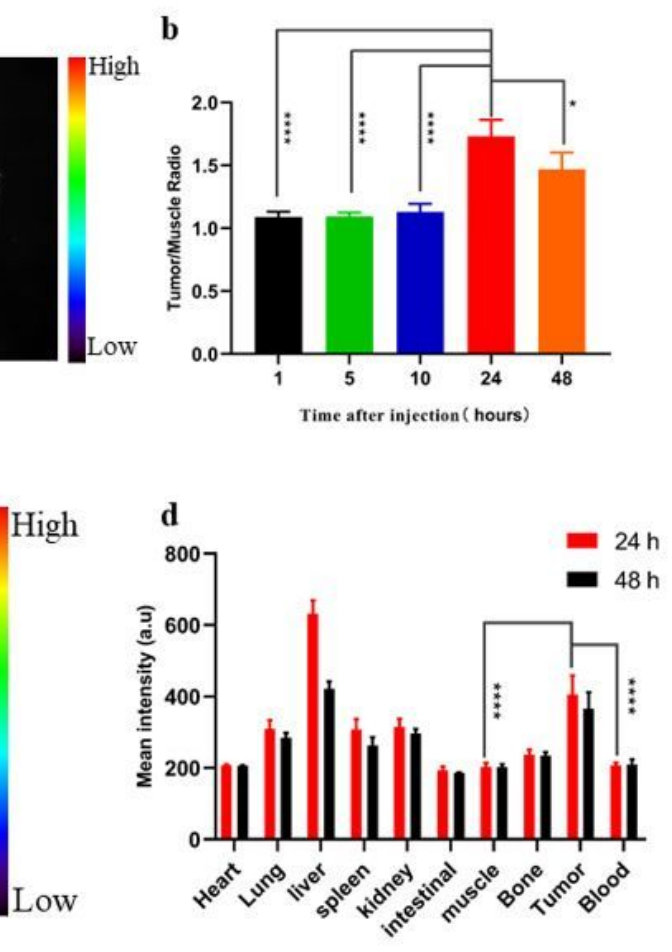

\section{Figure 4}

In vivo and ex vivo imaging with ICG-EM@ALA. In vivo fluorescence images (a) of 4T1 tumor-bearing mice taken at different time points and semi-quantitative analysis. (b). Ex vivo fluorescence images (c) of major organs and tumor dissected from mice at 24 hours and 48 hours and Semi-quantitative analysis (d). 
a

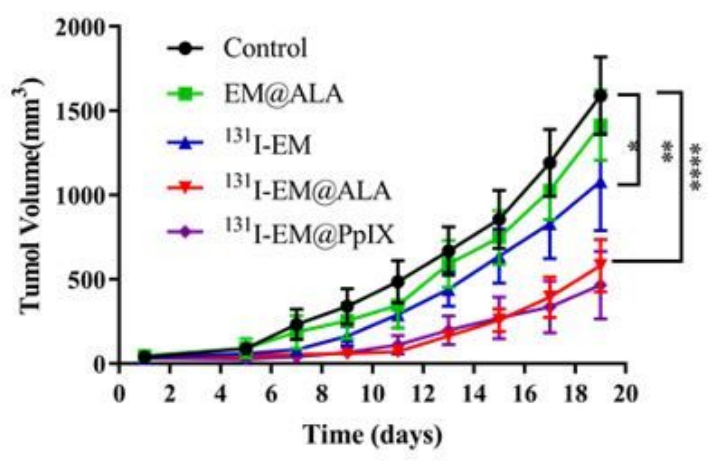

c

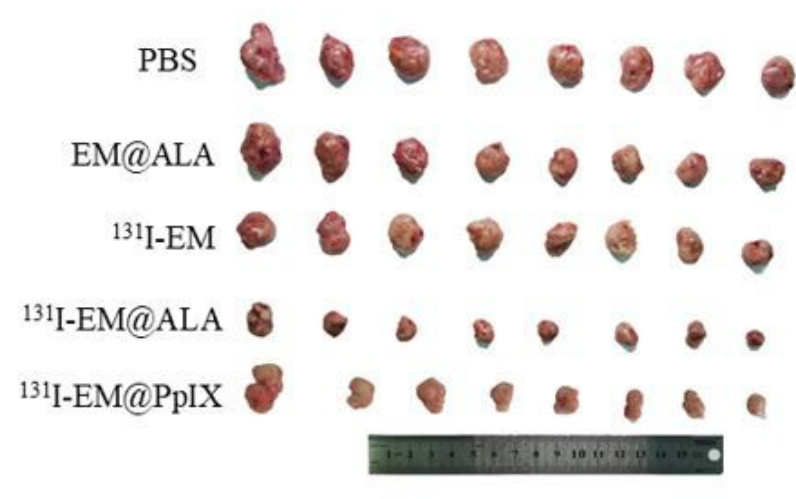

b

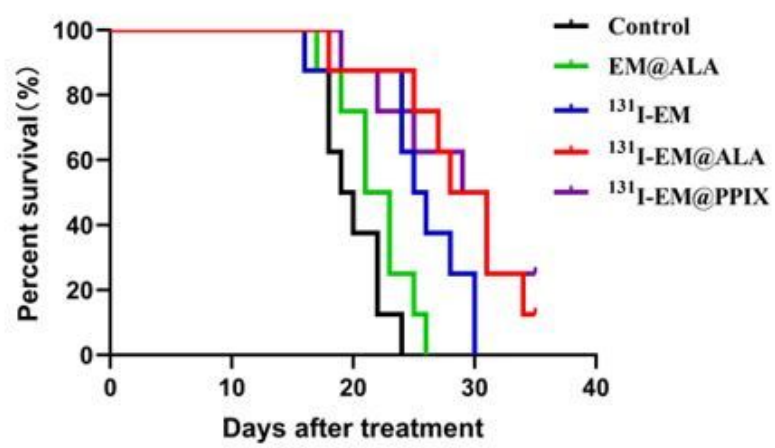

d

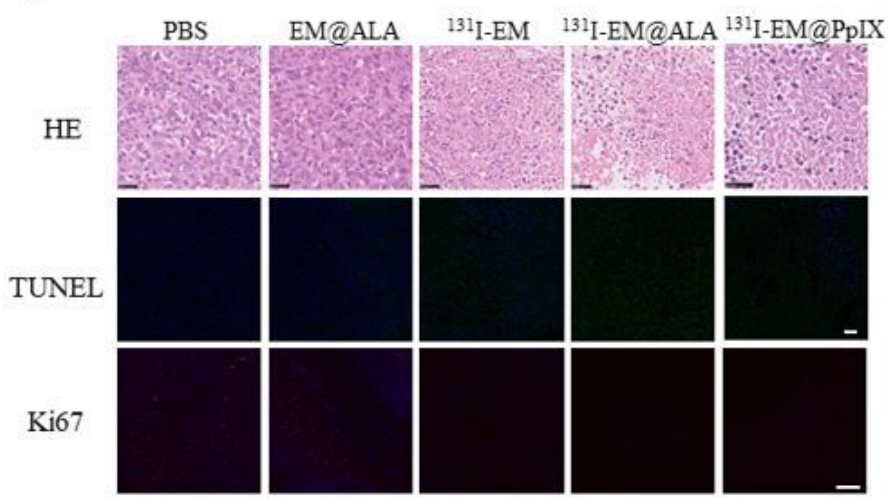

\section{Figure 5}

In vivo antitumor effects of ${ }^{131}$ I-EM@ALA. The tumor growth (a) and survival curves (b) of 4T1 tumors after different treatments. (c) Photographs of excised tumors at day 19. (d) HE staining of tumors. Scale bars $=25 \mu \mathrm{m}$. Ki67 staining and TENEL staining. Scale bars $=100 \mu \mathrm{m}$. 

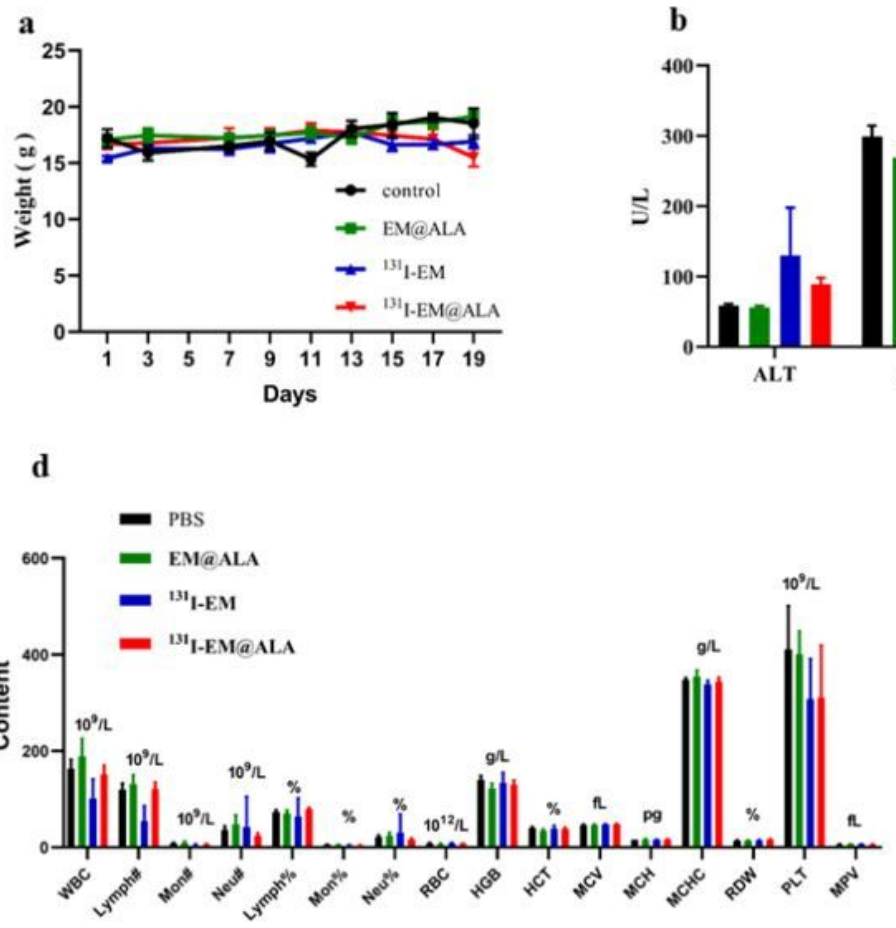
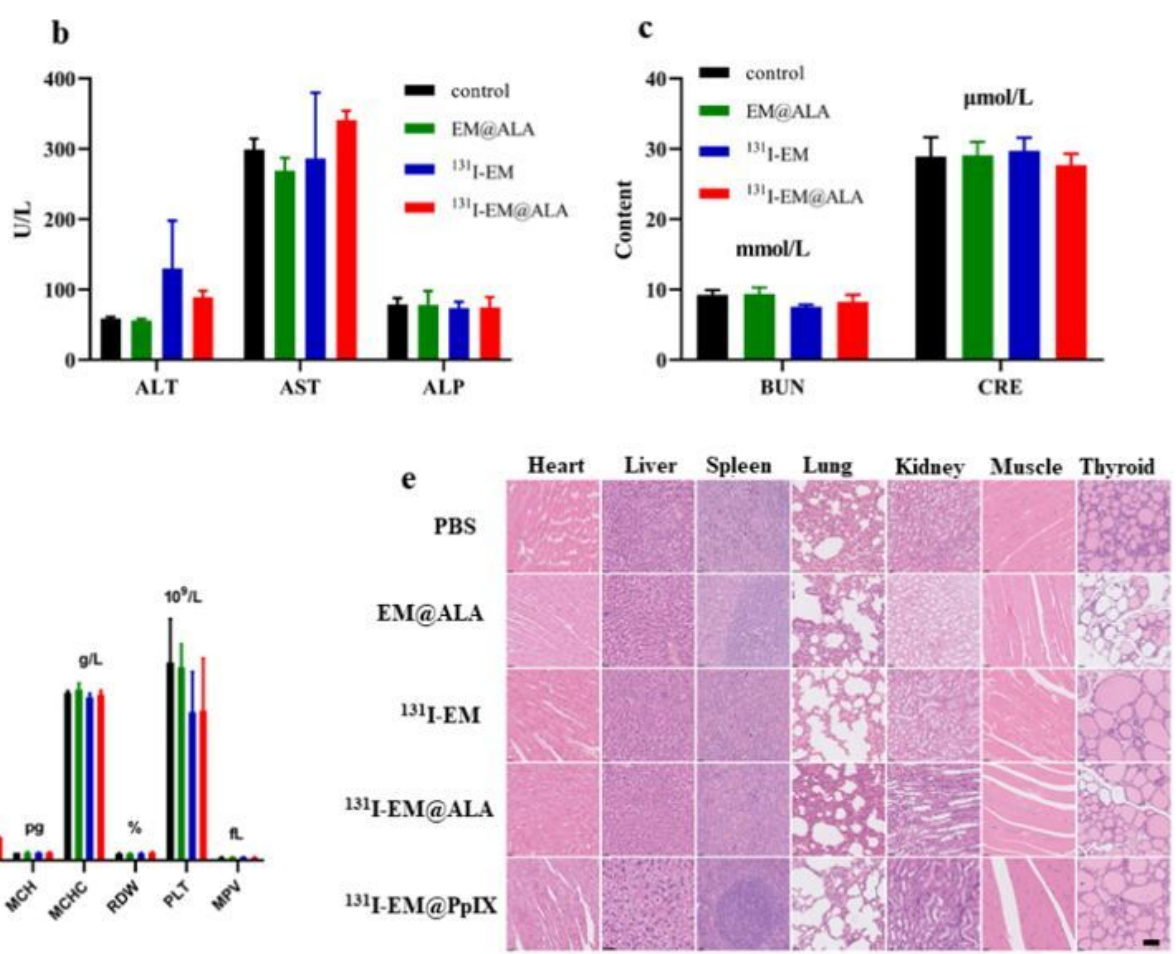

Figure 6

In vivo toxicity test. (a) Changes in animal body weight at 19 days. Blood biochemistry data including liver function markers (b): alanine aminotransferase (ALT), aspartate aminotransferase (AST) and alkaline phosphatase (ALP) and kidney function markers (c): blood urea nitrogen (BUN), creatinine (CRE). (d) Blood routine parameters data. White blood cells (WBC), lymphocytes number (Lymph\#), monocyte number (Mon\#), Neutrophil number (Neu\#), lymphocytes percentage (Lymph\%), monocyte percentage (Mon\%), neutrophil percentage (Neu\%), red blood cells (RBC), hemoglobin (HGB), hematocrit (HCT), mean corpuscular volume (MCV), mean corpuscular hemoglobin (MCH), and mean corpuscular hemoglobin concentration (MCHC), red cell volume distribution width (RDW), Platelets (PLT), mean platelet volume (MPV). (d) HE-stained slice images of major organs. Scale bars $=50 \mu \mathrm{m}$. 

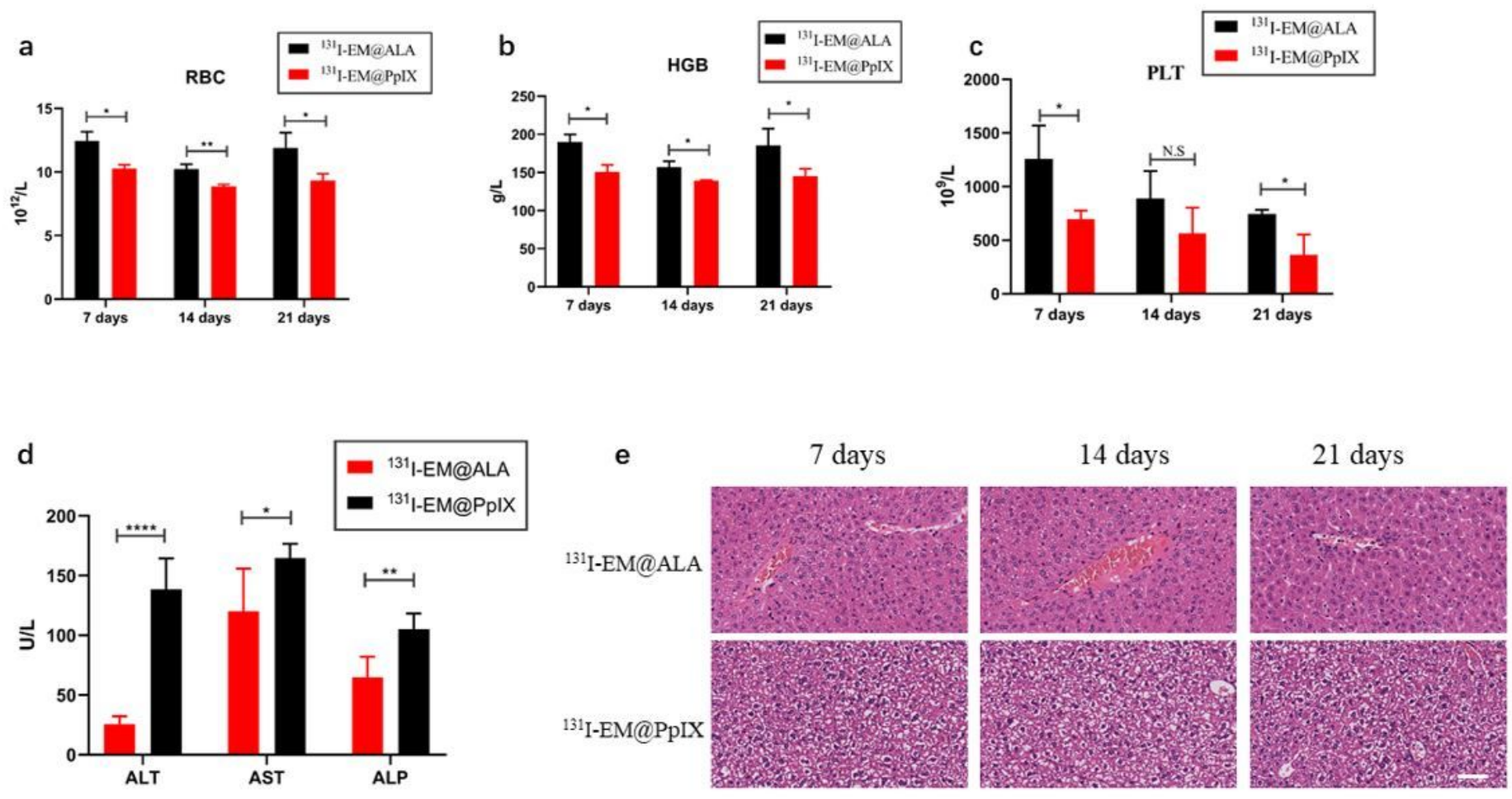

Figure 7

Toxicity comparison of two groups over time. (a, b, and c) RBC, PLT and HGB levels at 7 days,14 days, and 21 days. (d) AST, ALT, and ALP levels at 21 days. (e) HE-stained tissues from mice to monitor the histological changes in liver at different time points after intravenous injection of ${ }^{131}$ I-EM@ALA and ${ }^{131}$ IEM@PpIX. Scale bar: $100 \mu \mathrm{m}$. 
a

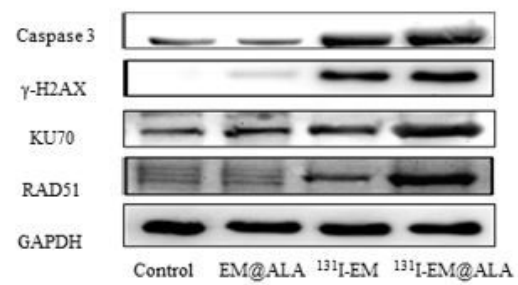

b
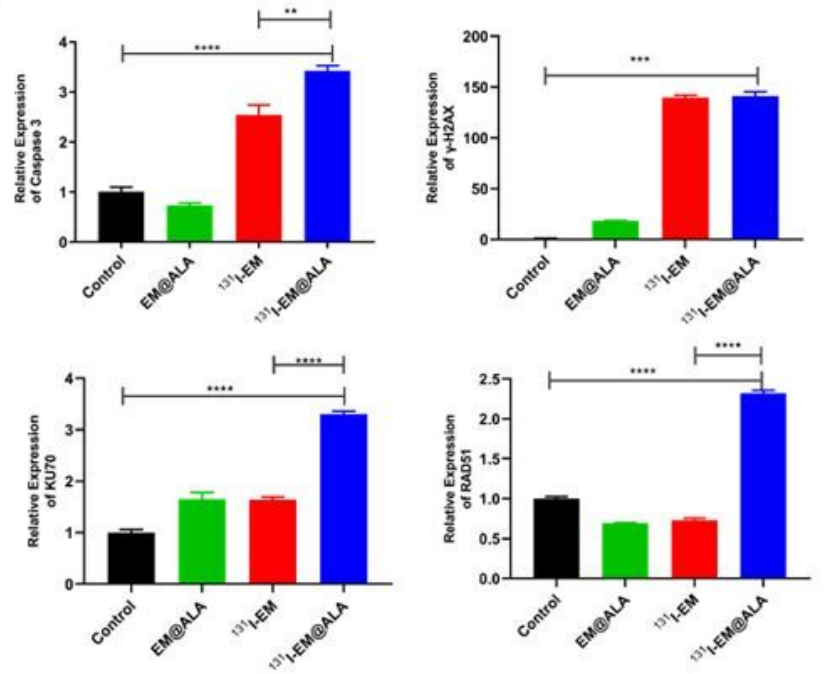

c

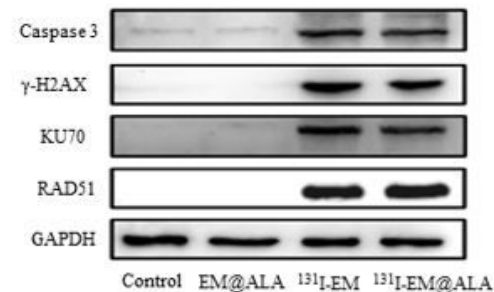

d
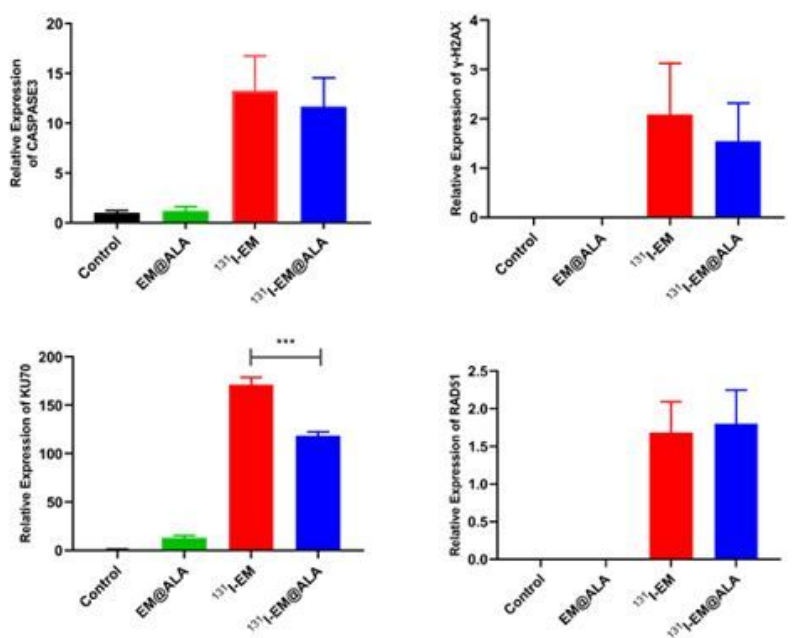

Figure 8

Effect of ${ }^{131} \mathrm{I}$-EM@ALA treatment on apoptotic-related proteins (caspase 3, $\mathrm{Y}-\mathrm{H} 2 \mathrm{AX}, \mathrm{KU70}$, and RAD51) in 4T1 cells determined by western blot in vitro (a) and in vivo (c). The relative protein expressions of caspase 3, $\mathrm{Y}-\mathrm{H} 2 \mathrm{AX}, \mathrm{KU} 70$, and RAD51. The protein expression levels were detected and evaluated in vitro (b) and in vivo (d) by Image J software, GAPDH was detected as a loading control.

\section{Supplementary Files}

This is a list of supplementary files associated with this preprint. Click to download.

- GraphicalAbstract.docx

- SupplementaryMaterial.docx 